Review began 08/31/2021 Review ended 09/08/2021 Published 09/16/2021

(c) Copyright 2021

Takakusagi et al. This is an open access article distributed under the terms of the Creative Commons Attribution License CCBY 4.0., which permits unrestricted use, distribution, and reproduction in any medium, provided the original author and source are credited.

\section{Comparison of Moderate Hypofractionated Volumetric-Modulated Arc Therapy Plans With and Without Flattening Filter for Localized Prostate Cancer}

Yosuke Takakusagi ${ }^{1}$, Keisuke Usui ${ }^{2}$, Nobutaka Mizoguchi ${ }^{1}$, Jun Nagatsuka ${ }^{3}$, Takeshi Hikage ${ }^{3}$, Yasuhiko Kodama $^{3}$, Takatomo Ezura ${ }^{4}$, Terufumi Kusunoki ${ }^{4}$, Yukio Oizumi ${ }^{5}$

1. Department of Radiation Oncology, Kanagawa Cancer Center, Yokohama, JPN 2. Department of Radiation Oncology, Juntendo University, Tokyo, JPN 3. Department of Radiology, Yokosuka General Hospital Uwamachi, Yokosuka, JPN 4. Section of Medical Physics and Engineering, Kanagawa Cancer Center, Yokohama, JPN 5. Department of Radiation Oncology, Yokosuka General Hospital Uwamachi, Yokosuka, JPN

Corresponding author: Yosuke Takakusagi, y-takakusagi@kcch.jp

\section{Abstract}

\section{Background/Aim}

The aim of this study was to compare volumetric-modulated arc therapy (VMAT) radiation plans between conventional VMAT with flattening filter (cFF-VMAT) and flattening filter-free VMAT (FFF-VMAT) for localized prostate cancer.

\section{Materials and methods}

Ten patients with localized prostate cancer who underwent cFF-VMAT at Yokosuka General Hospital Uwamachi, Yokosuka, Japan, from July 2020 to October 2020 were enrolled. Dose-volume histogram (DVH) parameters of the target volume, normal organs, monitor units (MU), and beam-on time (BOT) were compared between cFF-VMAT and FFF-VMAT plans.

\section{Results}

No significant difference was observed for DVH parameters for the target volume. No significant difference was observed in all parameters for the bladder and rectum between the CFF-VMAT and FFF-VMAT groups. The mean values of MU were $686 \pm 52$ and $784 \pm 80$ in cFF-VMAT and FFF-VMAT, respectively $(\mathrm{p}<0.001)$. The mean BOT was $97.0 \pm 6.6 \mathrm{~s}$ and $72.9 \pm 1.4 \mathrm{~s}$ for cFF-VMAT and FFF-VMAT, respectively $(\mathrm{p}<0.001)$.

\section{Conclusion}

DVH parameters of the target volume and normal organs were not significantly different between the cFFVMAT and FFF-VMAT plans. In FFF-VMAT, MU was significantly higher, and the BOT was significantly shorter than those in CFF-VMAT.

Categories: Radiation Oncology

Keywords: comparison, moderate hypofractionation, prostate cancer, vmat, flattening filter-free

\section{Introduction}

Prostate cancer is the second most common cancer worldwide and the fifth based on mortality rate [1]. Radiation therapy (RT) is one of the radical treatment modalities for localized or locally advanced prostate cancer. Favorable treatment outcomes have been reported by escalating the radiation dose [2-5]. Development of radiotherapy techniques, such as intensity-modulated radiotherapy (IMRT) and volumetricmodulated arc therapy (VMAT), can deliver dose escalation to the target volume without increasing the toxicity to surrounding normal organs [2]. Therefore, IMRT is widely used worldwide [6,7].

The $\alpha / \beta$ ratio of prostate cancer has been suggested to be very small [8-12]. Therefore, hypofractionated RT with a higher dose fraction was expected to demonstrate a favorable therapeutic effect. In fact, several randomized controlled trials on the use of hypofractionated RT for prostate cancer have been conducted and found to be comparable to the conventional fractionated RT [13-15]. Conversely, irradiation time generally becomes longer in hypofractionated RT. Intrafractional motion has been found to be increased as the irradiation time becomes longer [16].

Flattening filter-free (FFF) beams can provide higher dose rates and shorter irradiation times than flattening filter beams [17]. In several studies on FFF-VMAT for prostate cancers, FFF-VMAT has been reported to have similar dose distribution and shorter irradiation time compared to the conventional VMAT with flattening 
filter (cFF-VMAT) [18-21]. These studies demonstrated that ultrahypofractinated RT with a large dose fraction or conventional fractionation was applied for the treatment of prostate cancer. Although dose distribution and technical parameters of treatment may differ depending on dose fractionation, only rare studies have compared cFF-VMAT and FFF-VMAT in moderate hypofractionated RT for prostate cancer. Therefore, this study aimed to compare the moderate hypofractionated treatment plans of cFF-VMAT and FFF-VMAT for localized prostate cancer and quantitatively evaluate the dose distribution and technical parameters in irradiation.

\section{Materials And Methods \\ Patients}

This study enrolled 10 consecutive patients who underwent cFF-VMAT for localized or locally advanced prostate cancer at Yokosuka General Hospital Uwamachi, Yokosuka, Japan, from July 2020 to November 2020. The study was approved by the Institutional Review Board of Yokosuka General Hospital Uwamachi (approval number: 2020026).

\section{Treatment planning}

Patients were placed in the supine position and immobilized with heel support (Engineering System, Nagano, Japan). The patients urinated and drank water 60 min before undergoing computed tomography (CT) scan. CT was performed with 2-mm slices. Gross tumor volume was not delineated. Clinical target volume (CTV) included the entire prostate and proximal seminal vesicle. The planning target volume (PTV) was extended $5 \mathrm{~mm}$ in all directions from the CTV, except at 3-mm posterior margin. The rectum and bladder were delineated as organs at risk (OAR). The rectum was delineated from the anal canal to the rectosigmoid flexure. The same structure was used for cFF-VMAT and FFF-VMAT.

All treatment plans were analyzed retrospectively. The total dose was set at $70 \mathrm{~Gy}$ in 28 fractions and was prescribed as the mean dose (Dmean) of PTV [22]. Dose constraints for normal organs were as follows: percentage of the rectum volume receiving at least $37 \mathrm{~Gy}$ (V37) < 50\%, V60 < 18\% for rectum, and V60 < 40\% for bladder. Among these dose constraints, the equivalent dose in $2 \mathrm{~Gy}$ per fraction $(\alpha / \beta=3)$ was $32.0 \mathrm{~Gy}$ and $61.7 \mathrm{~Gy}$, respectively. Treatment plans were generated using Monaco version 5.11 (Elekta AB, Stockholm, Sweden). Treatment plans were created and reviewed by two or more radiation oncologists, radiation technologists, and medical physicists. To avoid bias in the creation and assessment of treatment plans, all treatment plans were created using the same plan template. A 10-MV (mega-electron-volt) $\mathrm{x}$-ray was used. The maximum dose rate was 600 monitor units (MU)/min for cFF-VMAT and $2200 \mathrm{MU} / \mathrm{min}$ for FFF-VMAT. VMAT plans used one full arc. The collimator angle, increment angle, and calculation grid were set at $10^{\circ}$, $30^{\circ}$, and $2 \mathrm{~mm}$, respectively. The calculation algorithm used was the x-ray voxel Monte Carlo method. Uncertainty per calculation was set at 1.0\%. All treatment plans were transferred to the MIM maestro software version 7.0 (MIM Software Inc., Cleveland, USA), and dose-volume histogram (DVH) parameters were estimated. The following DVH parameters were assessed: the dose covering $98 \%$ of the target volume (D98), D95, D50, D2, homogeneity index (HI), conformity index (CI), and mean absolute dose deviation (MADD) for PTV. HI was calculated using (D2-D98)/D50 [23]. CI was calculated using V95/VPTV [23]. MADD was calculated as:

$M A D D=\int_{0}^{V 0} \frac{|D-A|}{V 0} d V[24]$.

The notation was applied as follows: $\mathrm{D}$ is the dose, and $\mathrm{V}$ is the volume ordinate of the set of points representing the cumulative DVH curve of the structure, V0 is the volume of the structure, and A is the reference dose for the structure.

V95 represented the volume irradiated with $95 \%$ of the prescribed dose. VPTV represented the PTV volume. V10, V20, V30, V40, V50, V60, and Dmean were evaluated for the bladder and rectum.

MU and beam-on time (BOT) were investigated as technical parameters. The irradiation was performed using Axesse (Elekta AB, Stockholm, Sweden). BOT was measured by pressing the start button until the beam went off. BOT measurements were performed three times, and the average time was used.

\section{Statistical analysis}

DVH parameters for each treatment plan were compared using the Wilcoxon signed-rank test. The correlation between the PTV volume and technical parameters was evaluated using the Pearson correlation coefficient. A weak correlation was defined as $r<0.4$, moderate correlation as $0.4 \leqslant r \leqslant 0.7$, and strong correlation as $r>0.7$. A p-value of $<0.05$ was considered statistically significant. Statistical analysis was performed using the STATA software version 13.1 (StataCorp, College Station, USA).

\section{Results}

\section{Planning target volume}




\section{Cureus}

Figure 1 shows a typical dose distribution and DVH in the cFF-VMAT and FFF-VMAT plans. Figure 2 shows all DVH in the cFF-VMAT and FFF-VMAT plans. DVH parameters for PTV are summarized in Table 1. No significant difference was observed for DVH parameters in the cFF-VMAT and FFF-VMAT plans. The mean values of HI were $0.08 \pm 0.02$ and $0.08 \pm 0.02$ for cFF-VMAT and FFF-VMAT, respectively $(\mathrm{p}=0.170)$. The mean values of $\mathrm{CI}$ were $1.27 \pm 0.09$ and $1.25 \pm 0.08$ for cFF-VMAT and FFF-VMAT, respectively $(\mathrm{p}=0.981)$.

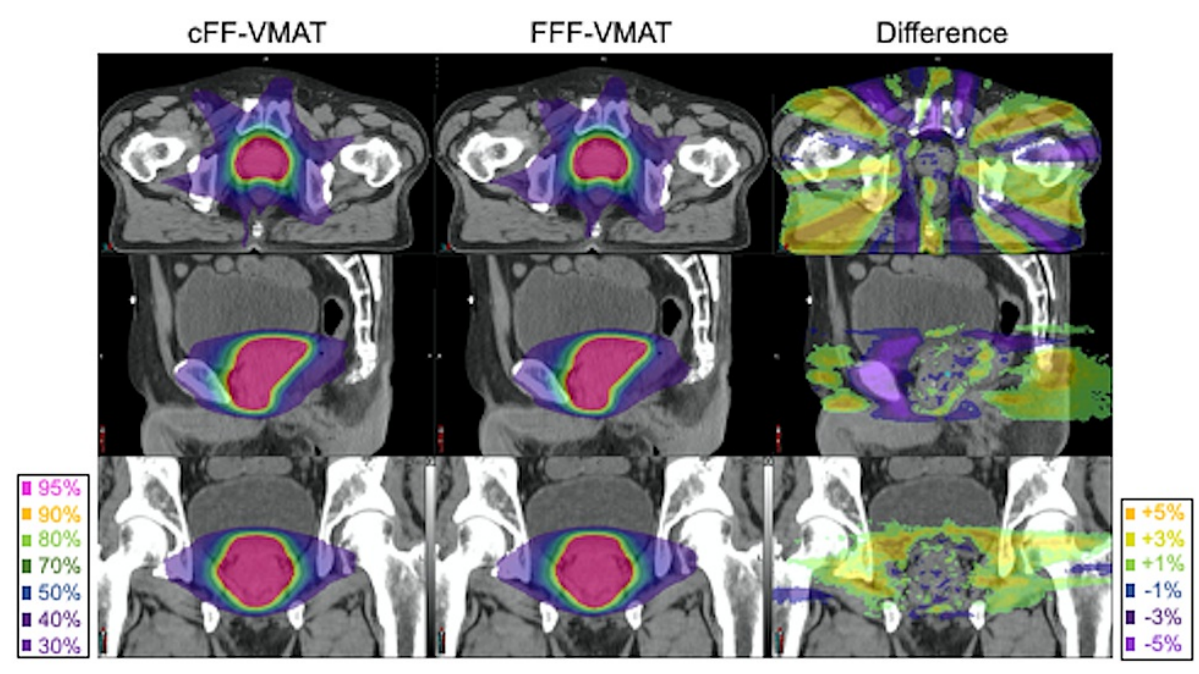

\section{FIGURE 1: Comparison of typical dose distribution}

The image shows the dose distributions of conventional volumetric-modulated arc therapy with flattening filter (cFF-VMAT) and VMAT with flattening filter-free beams (FFF-VMAT). The dose difference distribution map between cFF-VMAT and FFF-VMAT was also demonstrated.
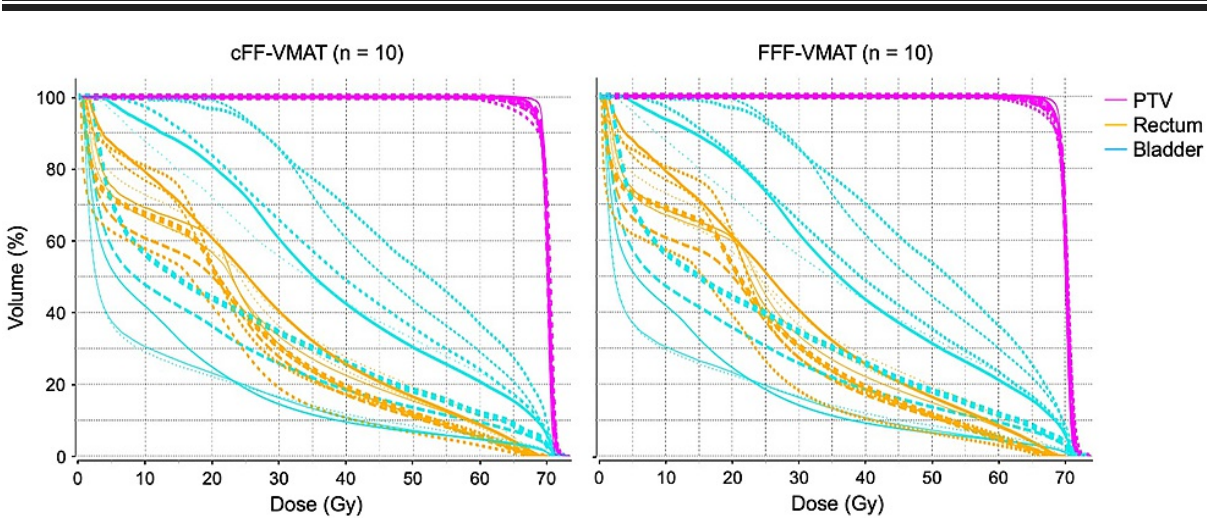

FIGURE 2: Dose-volume histogram of all cFF-VMAT and FFF-VMAT plans

VMAT, Volumetric-modulated arc therapy; cFF-VMAT, conventional volumetric-modulated arc therapy with flattening filter; FFF-VMAT, VMAT with flattening filter-free beams; PTV, planning target volume. 


\section{Cureus}

\begin{tabular}{|c|c|c|c|}
\hline DVH parameters & cFF-VMAT (mean \pm SD) & FFF-VMAT (mean \pm SD) & p-value \\
\hline \multicolumn{4}{|l|}{ PTV } \\
\hline D98 (Gy) & $66.2 \pm 1.6$ & $66.2 \pm 1.4$ & 0.647 \\
\hline D95 (Gy) & $68.1 \pm 0.7$ & $68.0 \pm 0.6$ & 0.541 \\
\hline D50 (Gy) & $70.2 \pm 0.1$ & $70.2 \pm 0.1$ & 0.799 \\
\hline D2 (Gy) & $71.5 \pm 0.2$ & $71.6 \pm 0.3$ & 0.146 \\
\hline Homogeneity index & $0.08 \pm 0.02$ & $0.08 \pm 0.02$ & 0.445 \\
\hline Conformity index & $1.27 \pm 0.09$ & $1.25 \pm 0.08$ & 0.058 \\
\hline MADD (Gy) & $0.38 \pm 0.09$ & $0.40 \pm 0.09$ & 0.525 \\
\hline
\end{tabular}

\section{TABLE 1: Dosimetric comparison for PTV between cFF-VMAT and FFF-VMAT}

VMAT, Volumetric-modulated arc therapy; cFF, conventional flattening filter; FFF, flattening filter-free; PTV, planning target volume; DVH, dose-volume histogram; MADD, mean absolute dose deviation; SD, standard deviation.

\section{Organs at risk}

DVH parameters for OARs are summarized in Table 2. No significant difference was observed in all parameters for the bladder and rectum between the cFF-VMAT and FFF-VMAT groups.

\begin{tabular}{|c|c|c|c|}
\hline DVH parameters & cFF-VMAT (mean \pm SD) & FFF-VMAT (mean \pm SD) & p-value \\
\hline \multicolumn{4}{|l|}{ Bladder } \\
\hline V10 (\%) & $68.4 \pm 29.7$ & $68.5 \pm 29.8$ & 0.838 \\
\hline V20 (\%) & $58.4 \pm 31.2$ & $58.5 \pm 31.4$ & 0.799 \\
\hline V30 (\%) & $46.1 \pm 28.0$ & $46.3 \pm 28.1$ & 0.508 \\
\hline V40 (\%) & $34.2 \pm 21.7$ & $34.4 \pm 22.0$ & 0.386 \\
\hline V50 (\%) & $25.0 \pm 16.4$ & $25.2 \pm 16.7$ & 0.285 \\
\hline V60 (\%) & $17.0 \pm 11.3$ & $17.0 \pm 11.3$ & 0.333 \\
\hline Dmean (Gy) & $29.7 \pm 14.7$ & $29.7 \pm 14.8$ & 1.000 \\
\hline \multicolumn{4}{|l|}{ Rectum } \\
\hline V10 (\%) & $69.7 \pm 7.6$ & $69.9 \pm 7.6$ & 0.386 \\
\hline V20 (\%) & $54.8 \pm 6.1$ & $55.7 \pm 7.2$ & 0.508 \\
\hline V30 (\%) & $31.3 \pm 6.4$ & $31.3 \pm 6.5$ & 0.959 \\
\hline V40 (\%) & $20.1 \pm 4.8$ & $20.1 \pm 5.0$ & 0.879 \\
\hline V50 (\%) & $13.1 \pm 3.6$ & $13.0 \pm 3.7$ & 0.241 \\
\hline V60 (\%) & $6.6 \pm 2.3$ & $6.3 \pm 2.4$ & 0.075 \\
\hline Dmean (Gy) & $23.9 \pm 2.9$ & $23.9 \pm 3.1$ & 0.476 \\
\hline
\end{tabular}

\section{TABLE 2: Dosimetric comparison for OARs between cFF-VMAT and FFF-VMAT}

VMAT, Volumetric-modulated arc therapy; cFF, conventional flattening filter; FFF, flattening filter-free; OAR, organs at risk; DVH, dose-volume histogram; SD, standard deviation. 


\section{Cureus}

\section{Technical parameters}

The technical parameters are summarized in Table 3. The mean PTV volume was $78.0 \pm 36.2 \mathrm{cc}$. The mean values of MU were $686 \pm 52$ and $784 \pm 80$ in cFF-VMAT and FFF-VMAT, respectively. MU was significantly higher in FFF-VMAT than that in cFF-VMAT $(\mathrm{p}<0.001)$. MU and PTV volumes were strongly correlated in both the cFF-VMAT and FFF-VMAT groups $(r=0.900$ and 0.930 , respectively). The mean percentage of MU increase was $14.2 \pm 4.0 \%$ in the FFF-VMAT plans compared to cFF-VMAT. The percentage of MU increase was moderately correlated with the PTV volume $(r=0.680)$ as shown in Figure 3.

\begin{tabular}{|c|c|c|}
\hline Parameters & Mean \pm SD & Pearson's r with PTV volume \\
\hline \multicolumn{3}{|l|}{ MU } \\
\hline cFF-VMAT & $686 \pm 52$ & 0.900 \\
\hline FFF-VMAT & $784 \pm 80$ & 0.930 \\
\hline MU increase (\%) & $14.2 \pm 4.0$ & 0.680 \\
\hline \multicolumn{3}{|l|}{ BOT } \\
\hline cFF-VMAT (sec) & $97.0 \pm 6.6$ & 0.850 \\
\hline FFF-VMAT (sec) & $72.9 \pm 1.4$ & 0.919 \\
\hline BOT decrease (\%) & $24.6 \pm 3.8$ & 0.740 \\
\hline
\end{tabular}

\section{TABLE 3: Pearson's correlation coefficient with PTV volume}

PTV, Planning target volume; MU, monitor unit; BOT, beam-on time; VMAT, volumetric-modulated arc therapy; cFF, conventional flattening filter; FFF, flattening filter-free; SD, standard deviation. 


\section{Cureus}

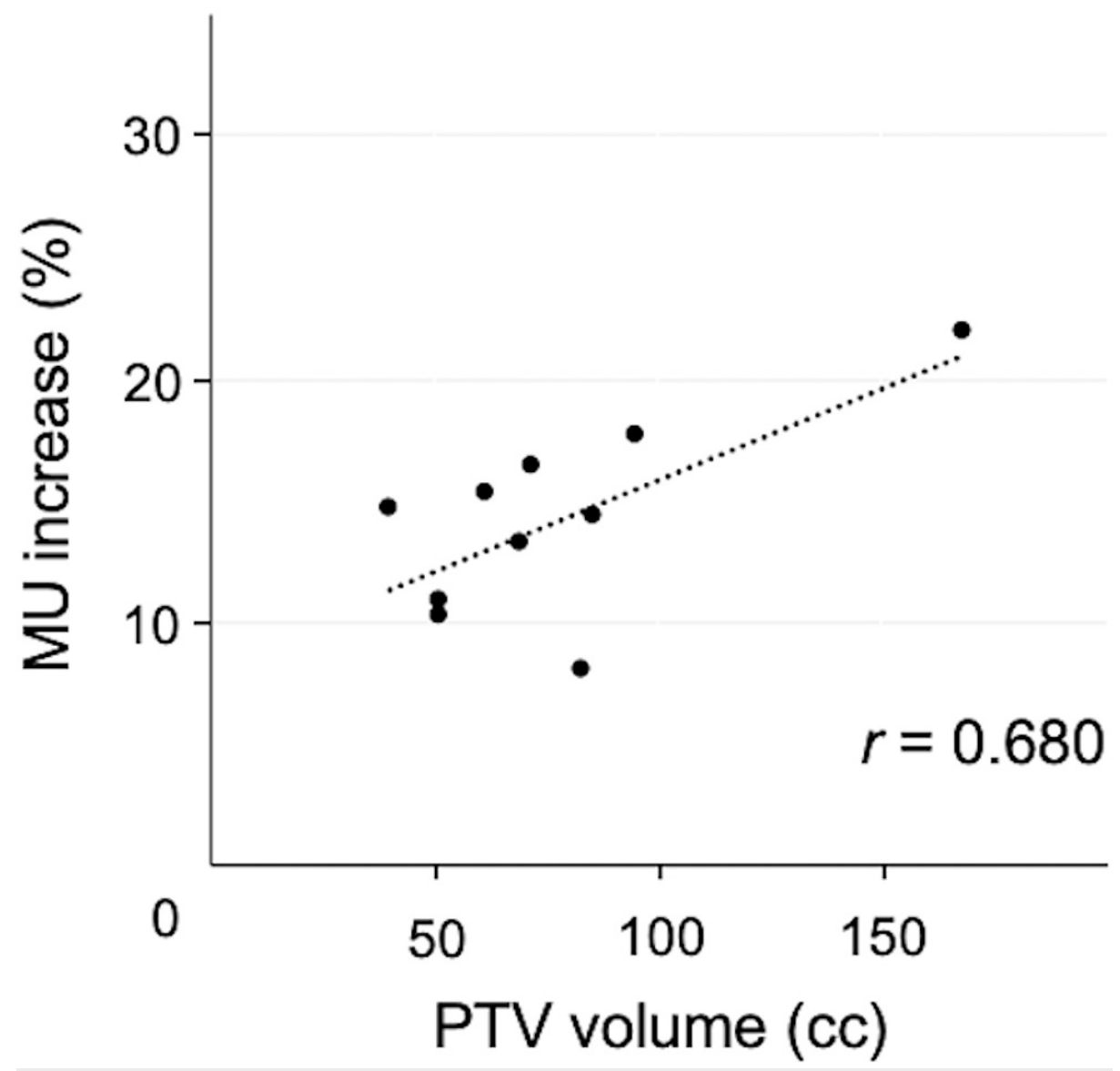

FIGURE 3: Scatter plots of planning target volume (PTV) volume and monitor units (MU) in volumetric-modulated arc therapy with flattening filter-free beams (FFF-VMAT)

The correlation between PTV volume and technical parameters was evaluated by the Pearson correlation coefficient. The PTV volume and percentage of MU increases are moderately correlated $(r=0.680)$.

The mean BOT was $97.0 \pm 6.6 \mathrm{~s}$ and $72.9 \pm 1.4 \mathrm{~s}$ for cFF-VMAT and FFF-VMAT, respectively. The BOT was significantly shorter in FFF-VMAT than that in cFF-VMAT $(\mathrm{p}<0.001)$.

The BOT and PTV volumes were strongly correlated in both the cFF-VMAT and FFF-VMAT groups $(r=0.850$ and 0.919 , respectively). The mean percentage of BOT decrease was $24.6 \pm 3.8 \%$ in the FFF-VMAT plans compared to cFF-VMAT. The percentage of BOT decrease was strongly correlated with PTV volume $(\mathrm{r}=$ 0.740 ) as shown in Figure 4. 


\section{Cureus}

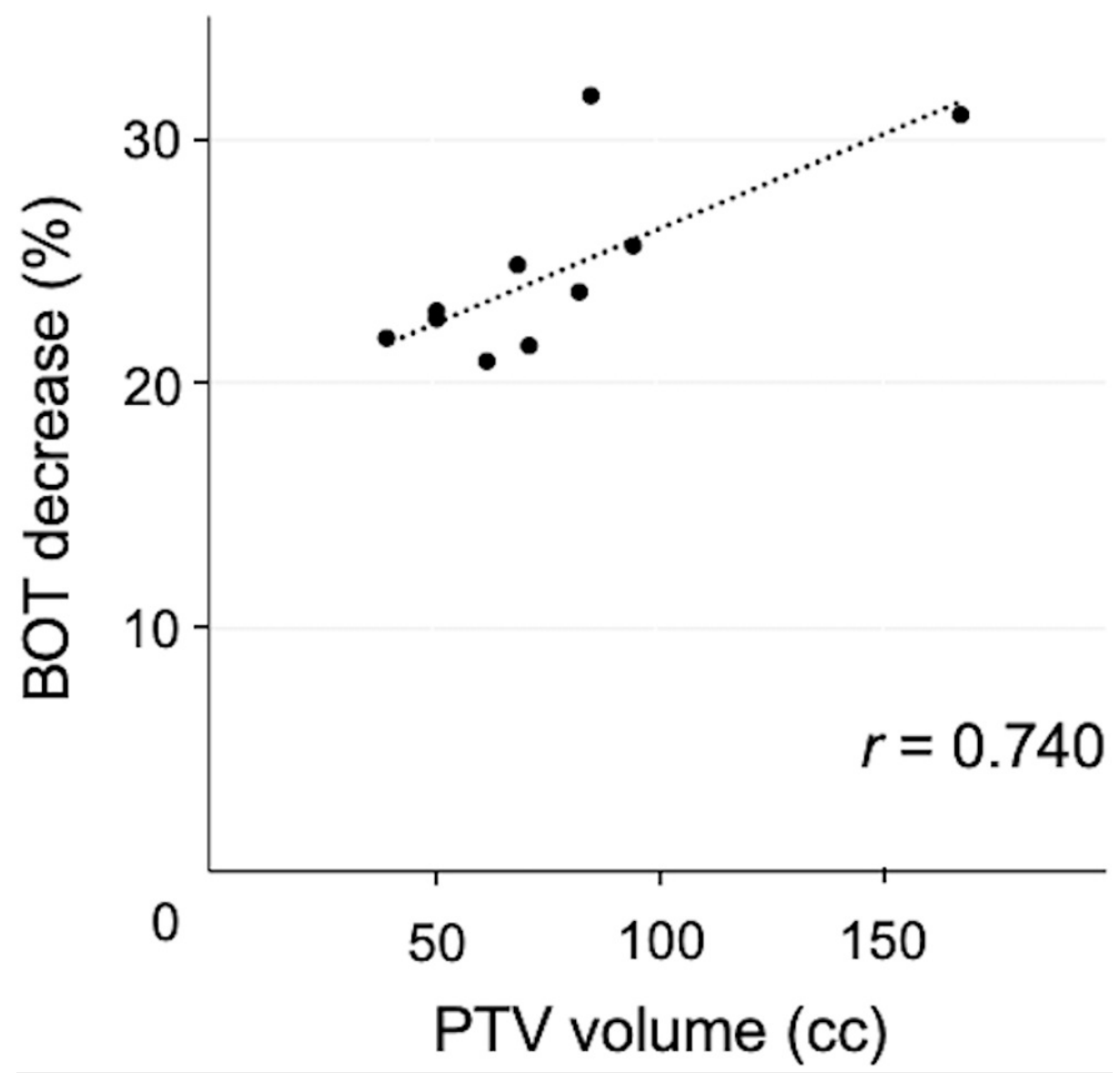

FIGURE 4: Scatter plots of planning target volume (PTV) and beam-on time (BOT) in volumetric-modulated arc therapy with flattening filter-free beams (FFF-VMAT)

The correlation between PTV volume and technical parameters was evaluated by the Pearson correlation coefficient. The PTV volume and percentage of BOT decrease were strongly correlated $(r=0.740)$.

\section{Discussion}

In this study, we compared cFF-VMAT and FFF-VMAT treatment plans for prostate cancer. All DVH parameters of PTV and OARs were not significantly different. In FFF-VMAT, MU was significantly higher, and the BOT was significantly shorter than those in cFF-VMAT. This is a rare report comparing cFF-VMAT and FFF-VMAT in moderate hypofractionated RT for prostate cancer.

Several studies have demonstrated that the difference of dose distribution in the target volume was not significant between FFF-VMAT and cFF-VMAT. Stereotactic RT using FFF-beam for lung cancer with a large dose fraction of 10-12.5 Gy showed that the dose distribution for target volume was comparable to the RT plan with conventional flattening filter beam [25-27]. PTV coverage was also comparable in a study of FFFVMAT for nasopharyngeal cancer [28]. A study of multiple treatment sites revealed that D2 and HI of PTV were significantly higher in FFF-VMAT than those in cFF-VMAT, but they were negligible [29]. Similar results have been reported for FFF-VMAT for prostate cancer in some studies. In studies on the use of ultrahypofractionated FFF-VMAT for prostate cancer, the CI and HI of the target volume were shown to be similar to that of the cFF-VMAT plan [18,19]. In addition, the target volume coverage was also comparable between cFF-VMAT and FFF-VMAT in the conventional fraction for prostate cancer [20]. In the present study using moderate hypofractionated irradiation, an equivalent dose distribution for PTV was obtained from each treatment group. Therefore, it was suggested that the dose distribution in the target volume was generally equivalent in FFF-VMAT, regardless of the treatment site or the dose fractionation.

Some studies demonstrated that DVH parameters of OARs were not also different between FFF-VMAT and cFF-VMAT. In a study of stereotactic RT using FFF-VMAT for lung cancer, doses in the lung, spinal cord, and heart were not different compared with cFF-VMAT [27]. A study of FFF-VMAT for prostate, head and neck, and brain cancers demonstrated similar doses of OARs with cFF-VMAT [29]. In addition, comparable dose distribution on OARs was observed with FFF-VMAT for prostate cancer [18-20], which was consistent with 
that of our study. Conversely, in FFF-VMAT of nasopharyngeal carcinoma, doses for the brainstem, spinal cord, and salivary glands were significantly higher than those in cFF-VMAT [28]. Therefore, several studies have demonstrated that FFF-VMAT generally provides a similar dose distribution for OARs to cFF-VMAT; however, there may be differences depending on the treatment site, and further study is warranted.

Several studies have shown that MU is higher in FFF-VMAT than that in cFF-VMAT. Additional MU is necessary to gain a uniform dose distribution with an inhomogeneous beam profile without FF [20]. A study on stereotactic RT for lung cancer showed that MU was increased by $5 \%$ with FFF-VMAT as compared to cFFVMAT [27]. In a study of nasopharyngeal carcinoma, MU was increased by $7 \%$ with FFF-VMAT as compared to cFF-VMAT [28]. MU was elevated by $10 \%-25 \%$ in FFF-VMAT as compared to cFF-VMAT in prostate cancer [18-21]. The present study also showed similar results, i.e., MU values were significantly higher in FFF-VMAT and increased by $14.2 \%$ than cFF-VMAT. Hence, it was suggested that MU was higher in FFF-VMAT than that in cFF-VMAT, regardless of the treatment site or the dose fractionation.

Arslan et al. compared cFF-IMRT and FFF-IMRT according to the PTV volume [30]. MU was significantly higher in the larger PTV volume group than that in the smaller PTV volume. In that study, comparable dose distribution and higher MU were also demonstrated in FFF-IMRT. Vieillevigne et al. reported on the effect of PTV volume on cFF-VMAT and FFF-VMAT to a virtual phantom [31]. They showed that an increase in PTV volume was significantly correlated with the percentage of MU increase in FFF-VMAT. The present study also demonstrated a moderate correlation between PTV volume and percentage of MU increase. Although MU was suggested to be higher in patients with larger PTV volume, reports on the relationship between PTV volume and MU in FFF-beam are still limited, and further studies are needed.

One of the advantages of FFF-VMAT is shortening the treatment time. In stereotactic RT for lung cancer, FFF-VMAT reduced the irradiation time by approximately $50 \%-80 \%$ than cFF-VMAT [26,27,32]. In the treatment of nasopharyngeal carcinoma using a total dose of $70 \mathrm{~Gy}$ in 31 fractions, the irradiation time was not reduced [28]. In that study, according to the small dose fraction delivered with two full arcs of beams, the irradiation time was speculated to be limited by the gantry rotation and movement speed of the multi-leaf collimator. In addition, the large target volume for nasopharyngeal cancer may have also affected the irradiation time. Several studies of FFF-VMAT for prostate cancer reported an 8\%-25\% reduction in irradiation time [18-21]. Similar results were obtained in the present study, where FFF-VMAT reduced the BOT by $24.6 \%$. Intrafractional motion cannot be ignored in the RT for prostate cancer [20]. Therefore, it is expected that intrafractional motion can be suppressed by shortening the irradiation time in prostate cancer by the use of FFF-VMAT. In addition, shortening the treatment time is patient-friendly and is also expected to improve the treatment throughput.

There are several limitations of this study. First, this study was a small-number survey in a single facility. Second, we investigated only the Monaco treatment planning system. In addition, various VMAT plan parameters, such as statistical uncertainty, minimum segment width, and fluence smoothing, can be modified in the Monaco treatment planning system. Several studies suggested that MU and irradiation time can vary by adjusting these parameters [33-35]. Several anatomical factors such as the size of the target volume and location of OARs may affect dose distribution and MU. Further studies are needed to clarify the similarities and differences between FFF-VMAT and cFF-VMAT.

\section{Conclusions}

In this study, we compared the RT plans between cFF-VMAT and FFF-VMAT for prostate cancer. The dose distribution of FFF-VMAT was comparable to that of cFF-VMAT. The higher MU and shorter BOT were shown in FFF-VMAT compared to cFF-VMAT.

\section{Additional Information \\ Disclosures}

Human subjects: Consent was obtained or waived by all participants in this study. Institutional Review Board of Yokosuka General Hospital Uwamachi issued approval 2020026. Animal subjects: All authors have confirmed that this study did not involve animal subjects or tissue. Conflicts of interest: In compliance with the ICMJE uniform disclosure form, all authors declare the following: Payment/services info: All authors have declared that no financial support was received from any organization for the submitted work. Financial relationships: All authors have declared that they have no financial relationships at present or within the previous three years with any organizations that might have an interest in the submitted work. Other relationships: All authors have declared that there are no other relationships or activities that could appear to have influenced the submitted work.

\section{References}

1. Bray F, Ferlay J, Soerjomataram I, Siegel RL, Torre LA, Jemal A: Global cancer statistics 2018: GLOBOCAN estimates of incidence and mortality worldwide for 36 cancers in 185 countries. CA Cancer J Clin. 2018, 68:394-424. 10.3322/caac.21492

2. Sanguineti G, Cavey ML, Endres EJ, Brandon GG, Bayouth JE: Is IMRT needed to spare the rectum when 
pelvic lymph nodes are part of the initial treatment volume for prostate cancer?. Int J Radiat Oncol Biol Phys. 2006, 64:151-60. 10.1016/j.ijrobp.2005.06.026

3. Lyons JA, Kupelian PA, Mohan DS, Reddy CA, Klein EA: Importance of high radiation doses (72 Gy or greater) in the treatment of stage T1-T3 adenocarcinoma of the prostate. Urology. 2000, 55:85-90. 10.1016/s0090-4295(99)00380-5

4. Kupelian PA, Mohan DS, Lyons J, Klein EA, Reddy CA: Higher than standard radiation doses (> or =72 Gy) with or without androgen deprivation in the treatment of localized prostate cancer. Int J Radiat Oncol Biol Phys. 2000, 46:567-574. 10.1016/s0360-3016(99)00455-1

5. Kupelian PA, Buchsbaum JC, Reddy CA, Klein EA: Radiation dose response in patients with favorable localized prostate cancer (Stage T1-T2, biopsy Gleason < or =6, and pretreatment prostate-specific antigen < or = 10). Int J Radiat Oncol Biol Phys. 2001, 50:621-625. 10.1016/s0360-3016(01)01466-3

6. Takeda K, Takai Y, Narazaki K, et al.: Treatment outcome of high-dose image-guided intensity-modulated radiotherapy using intra-prostate fiducial markers for localized prostate cancer at a single institute in Japan. Radiat Oncol. 2012, 7:105. 10.1186/1748-717X-7-105

7. Zelefsky MJ, Levin EJ, Hunt M, Yamada Y, Shippy AM, Jackson A, Amols HI: Incidence of late rectal and urinary toxicities after three-dimensional conformal radiotherapy and intensity-modulated radiotherapy for localized prostate cancer. Int J Radiat Oncol Biol Phys. 2008, 70:1124-9. 10.1016/j.ijrobp.2007.11.044

8. Sanfilippo NJ, Cooper BT: Hypofractionated radiation therapy for prostate cancer: biologic and technical considerations. Am J Clin Exp Urol. 2014, 2:286-293.

9. Fowler JF, Ritter MA, Chappell RJ, et al.: What hypofractionated protocols should be tested for prostate cancer?. Int J Radiat Oncol Biol Phys. 2003, 56:1093-1104. 10.1016/s0360-3016(03)00132-9

10. Brenner DJ, Martinez AA, Edmundson GK, Mitchell C, Thames HD, Armour EP: Direct evidence that prostate tumors show high sensitivity to fractionation (low alpha/beta ratio), similar to late-responding normal tissue. Int J Radiat Oncol Biol Phys. 2002, 52:6-13. 10.1016/s0360-3016(01)02664-5

11. D’Souza WD, Thames HD: Is the alpha/beta ratio for prostate cancer low? . Int J Radiat Oncol Biol Phys. 2001, 51:1-3. 10.1016/s0360-3016(01)01650-9

12. King CR, Fowler JF: A simple analytic derivation suggests that prostate cancer alpha/beta ratio is low . Int J Radiat Oncol Biol Phys. 2001, 51:213-214. 10.1016/s0360-3016(01)01651-0

13. Dearnaley D, Syndikus I, Mossop H, et al.: Conventional versus hypofractionated high-dose intensitymodulated radiotherapy for prostate cancer: 5 -year outcomes of the randomised, non-inferiority, phase 3 CHHiP trial. Lancet Oncol. 2016, 17:1047-60. 10.1016/S1470-2045(16)30102-4

14. Catton CN, Lukka H, Gu CS, et al.: Randomized trial of a hypofractionated radiation regimen for the treatment of localized prostate cancer. J Clin Oncol. 2017, 35:1884-90. 10.1200/JCO.2016.71.7397

15. Widmark A, Gunnlaugsson A, Beckman L, et al.: Ultra-hypofractionated versus conventionally fractionated radiotherapy for prostate cancer: 5-year outcomes of the HYPO-RT-PC randomised, non-inferiority, phase 3 trial. Lancet. 2019, 394:385-395. 10.1016/S0140-6736(19)31131-6

16. Kupelian P, Willoughby T, Mahadevan A, et al.: Multi-institutional clinical experience with the Calypso System in localization and continuous, real-time monitoring of the prostate gland during external radiotherapy. Int J Radiat Oncol Biol Phys. 2007, 67:1088-98. 10.1016/j.ijrobp.2006.10.026

17. Xiao Y, Kry SF, Popple R, et al.: Flattening filter-free accelerators: a report from the AAPM Therapy Emerging Technology Assessment Work Group. J Appl Clin Med Phys. 2015, 16:5219. 10.1120/jacmp.v16i3.5219

18. Chung JB, Kim JS, Eom KY, et al.: Comparison of VMAT-SABR treatment plans with flattening filter (FF) and flattening filter-free (FFF) beam for localized prostate cancer. J Appl Clin Med Phys. 2015, 16:302-313. 10.1120/jacmp.v16i6.5728

19. Zwahlen DR, Lang S, Hrbacek J, et al.: The use of photon beams of a flattening filter-free linear accelerator for hypofractionated volumetric modulated arc therapy in localized prostate cancer. Int J Radiat Oncol Biol Phys. 2012, 83:1655-60. 10.1016/j.ijrobp.2011.10.019

20. Treutwein M, Hipp M, Koelbl O, Dobler B: Volumetric-modulated arc therapy and intensity-modulated radiation therapy treatment planning for prostate cancer with flattened beam and flattening filter free linear accelerators. J Appl Clin Med Phys. 2017, 18:307-14. 10.1002/acm2.12168

21. Lechner W, Kragl G, Georg D: Evaluation of treatment plan quality of IMRT and VMAT with and without flattening filter using Pareto optimal fronts. Radiother Oncol. 2013, 109:437-41. 10.1016/j.radonc.2013.09.020

22. Kupelian PA, Thakkar VV, Khuntia D, Reddy CA, Klein EA, Mahadevan A: Hypofractionated intensitymodulated radiotherapy (70 gy at $2.5 \mathrm{~Gy}$ per fraction) for localized prostate cancer: long-term outcomes. Int J Radiat Oncol Biol Phys. 2005, 63:1463-8. 10.1016/j.ijrobp.2005.05.054

23. Hodapp N: [The ICRU Report 83: prescribing, recording and reporting photon-beam intensity-modulated radiation therapy (IMRT)]. Strahlenther Onkol. 2012, 188:97-9. 10.1007/s00066-011-0015-X

24. Wang X, Fargier-Bochaton O, Dipasquale G, et al.: Is prone free breathing better than supine deep inspiration breath-hold for left whole-breast radiotherapy? A dosimetric analysis. Strahlenther Onkol. 2021, 197:317-31. 10.1007/s00066-020-01731-8

25. Vassiliev ON, Kry SF, Chang JY, Balter PA, Titt U, Mohan R: Stereotactic radiotherapy for lung cancer using a flattening filter free Clinac. J Appl Clin Med Phys. 2009, 10:14-21. 10.1120/jacmp.v10i1.2880

26. Navarria P, Ascolese AM, Mancosu P, et al.: Volumetric modulated arc therapy with flattening filter free (FFF) beams for stereotactic body radiation therapy (SBRT) in patients with medically inoperable early stage non small cell lung cancer (NSCLC). Radiother Oncol. 2013, 107:414-8. 10.1016/j.radonc.2013.04.016

27. Hrbacek J, Lang S, Graydon SN, Klöck S, Riesterer O: Dosimetric comparison of flattened and unflattened beams for stereotactic ablative radiotherapy of stage I non-small cell lung cancer. Med Phys. 2014, 41:031709. 10.1118/1.4866231

28. Zhuang M, Zhang T, Chen Z, et al.: Volumetric modulation arc radiotherapy with flattening filter-free beams compared with conventional beams for nasopharyngeal carcinoma: a feasibility study. Chin J Cancer. 2013, 32:397-402. 10.5732/cjc.012.10182

29. Ronaldson JP, Bennett H, Roberts J, Ronaldson AJ, Cousins AT: A dosimetric comparison of flattening filter 


\section{Cureus}

free and conventional VMAT treatments for some common cancer sites. Phys Eng Sci Med. 2020, 43:719-25. 10.1007/s13246-020-00877-0

30. Arslan A, Sengul B: Comparison of radiotherapy techniques with flattening filter and flattening filter-free in lung radiotherapy according to the treatment volume size. Sci Rep. 2020, 10:8983. 10.1038/s41598-02066079-6

31. Vieillevigne L, Bessieres S, Ouali M, Lanaspeze C: Dosimetric comparison of flattened and unflattened beams for stereotactic body radiation therapy: impact of the size of the PTV on dynamic conformal arc and volumetric modulated arc therapy. Phys Med. 2016, 32:1405-14. 10.1016/j.ejmp.2016.10.007

32. Prendergast BM, Fiveash JB, Popple RA, et al.: Flattening filter-free linac improves treatment delivery efficiency in stereotactic body radiation therapy. J Appl Clin Med Phys. 2013, 14:4126. 10.1120/jacmp.v14i3.4126

33. Palanisamy M, David K, Durai M, Bhalla N, Puri A: Dosimetric impact of statistical uncertainty on Monte Carlo dose calculation algorithm in volumetric modulated arc therapy using Monaco TPS for three different clinical cases. Rep Pract Oncol Radiother. 2019, 24:188-99. 10.1016/j.rpor.2019.01.005

34. Wang Y, Chen L, Zhu F, Guo W, Zhang D, Sun W: A study of minimum segment width parameter on VMAT plan quality, delivery accuracy, and efficiency for cervical cancer using Monaco TPS. J Appl Clin Med Phys. 2018, 19:609-15. 10.1002/acm2.12422

35. Sarkar B, Manikandan A, Nandy M, Munshi A, Sayan P, Sujatha N: Influence of monte carlo variance with fluence smoothing in VMAT treatment planning with Monaco TPS. Indian J Cancer. 2016, 53:158-61. 10.4103/0019-509X.180820 